\title{
Efecto del dióxido de titanio en las propiedades mecánicas y autolimpiantes del mortero
}

\author{
Carlos Magno Chavarry Vallejos \\ ORCID: https://orcid.org/0000-0003-0512-8954 \\ carlos.chavarry@upc.pe \\ Universidad Peruana de Ciencias Aplicadas \\ Lima, Perú
}

\author{
Xavier Antonio Laos Laura \\ ORCID: https://orcid.org/0000-0003-0715-8348 \\ xavier.laos@upc.pe \\ Universidad Ricardo Palma \\ Lima, Perú
}

Enriqueta Pereyra Salardi

ORCID: https://orcid.org/0000-0003-2527-3665

enriqueta.pereyra@urp.edu.pe

Universidad Ricardo Palma

Lima, Perú

\author{
Liliana Janet Chavarría Reyes \\ ORCID: https://orcid.org/0000-0002-1759-2132 \\ liliana.chavarria@urp.edu.pe \\ Universidad Peruana de Ciencias Aplicadas \\ Lima, Perú
}

\author{
Andrés Avelino Valencia Gutiérrez \\ ORCID: https://orcid.org/0000-0002-8873-189X \\ avalenciag@usmp.pe \\ Universidad San Martín de Porres \\ Lima, Perú
}

Karen Romina Martínez Zuasnábar

ORCID: https://orcid.org/0000-0001-8645-9581

karen.martinez.zuasnábar.km@gmail.com

Universidad Ricardo Palma

Lima, Perú

Recibido (23/04/21), Aceptado (14/05/21)

\begin{abstract}
Resumen: El presente artículo tiene como objetivo determinar la influencia de la adición del dióxido de titanio (TiO2) en el mortero de cemento Pórtland Tipo I. La investigación es descriptiva, correlacional, explicativo, con diseño experimental, longitudinal, prospectivo y estudio de cohorte. Se elaboró una mezcla patrón y tres mezclas de mortero con $5 \%, 7.5 \%$ y $10 \%$ de contenido de $\mathrm{TiO} 2$ como reemplazo del volumen de cemento para las propiedades autolimpiantes se realizó el ensayo de rodamina e intemperismo. La incorporación de dióxido de titanio disminuyó la resistencia a la compresión, incrementó la fluidez y tasa de absorción de agua; la prueba de rodamina dio que el mortero sin actividad fotocatalítico no contenía $\mathrm{TiO} 2$ porque no cumple con los factores de fotodegradación R4 y R26. Mediante la exposición de paneles al intemperismo favoreciendo la propiedad autolimpiante de los morteros con adición de $\mathrm{TiO} 2(5 \%)$.
\end{abstract}

Palabras Clave: Actividad foto catalítico, dióxido de titanio, factores de fotodegradación, propiedades mecánicas y autolimpiante.

\section{Effect of titanium dioxide on the mechanical and self-cleaning properties of mortar}

Abstract: The objective of this article is to determine the influence of the addition of titanium dioxide (TiO2) in Type I Portland cement mortar. The research is descriptive, correlational, explanatory, with an experimental, longitudinal, prospective design and a cohort study. A standard mixture and three mortar mixtures with $5 \%, 7.5 \%$ and $10 \% \mathrm{TiO} 2$ content were prepared as replacement of the cement volume for self-cleaning properties, the rhodamine and weathering test was carried out. The incorporation of titanium dioxide decreased compressive strength, increased fluidity and water absorption rate; the rhodamine test showed that the mortar without photocatalytic activity did not contain $\mathrm{TiO} 2$ because it does not comply with the photodegradation factors $\mathrm{R} 4$ and $\mathrm{R} 26$. By exposing panels to weathering, favoring the self-cleaning property of mortars with the addition of $\mathrm{TiO} 2(5 \%)$.

Keywords: Photocatalytic activity, photodegradation factors, titanium dioxide, mechanical properties and selfcleaning.

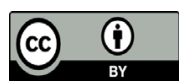




\section{Introducción}

La necesidad de nuevos materiales de construcción comprometidos con el medio ambiente ha dado lugar al uso de morteros fotocatalíticos, los cuales tienen un buen desempeño ante el deterioro de fachadas causado por intemperismo. Este tipo de morteros están siendo utilizados y evaluados en distintos países europeos debido a los resultados positivos que se están obteniendo. En el ámbito ambiental, el proceso de fotocatálisis en morteros adicionados con $\mathrm{TiO} 2$ permite reducir un determinado rango de compuestos orgánicos e inorgánicos presentes en el aire. Su uso en fachadas contribuirá con la purificación del aire. El nivel de contaminación atmosférica en Lima Metropolitana registra una media anual de 38 microgramos de PM 2,5 por metro cúbico superando desde hace varios años al máximo establecido por la Organización Mundial de la Salud siendo el permitido 10 microgramos, debido a este indicador Lima Metropolitana es considerada una de las ciudades con indicadores de calidad de aire muy bajas en América Latina. Estos contaminantes atmosféricos no sólo provocan daños a la salud o al medio ambiente, sino que también contribuyen al deterioro de las fachadas de las construcciones, aumentando los costos de mantenimiento y rehabilitación. En la búsqueda de colaboración del sector de construcción hacia la preservación del medio ambiente se vienen desarrollando materiales con propiedades autolimpiantes y descontaminantes otorgados por adiciones de fotocatalizadores como es el dióxido de titanio ( $\mathrm{TiO} 2)$. Asimismo, existen investigaciones donde se ha demostrado la degradación de compuestos orgánicos e inorgánicos presentes en el aire en materiales a base de cemento con adición de TiO2. El uso de morteros de cemento con adición de dióxido de titanio puede ser una alternativa para prolongar el tiempo de vida de las fachadas y a su vez contribuir con la reducción de la contaminación atmosférica. Sin embargo, al ser una tecnología nueva existe cierto desconocimiento en el efecto que produce esta adición en las propiedades del mortero, así como también el porcentaje adecuado a utilizar y falta de información sobre la normativa vigente.

El estudio realizado por Medina en el año 2017, tuvo como objetivo determinar la influencia del uso del dióxido de titanio en las propiedades del mortero de cemento blanco y la variación de los costos de su elaboración y se basó en un estudio experimental sobre cuatro tratamientos de morteros con distintos porcentajes de dióxido de titanio, para evaluar sus propiedades mecánicas [1]. Los ensayos realizados fueron de absorción, fluidez, resistencia a la compresión, propiedad autolimpiable y costos. Abella en el año 2015 realizó el estudio de los mecanismos de activación fotocatalítica en concentraciones de $\mathrm{TiO} 2$ en los materiales a base de cemento, su comportamiento hidrófobo, rendimientos autolimpiantes y descontaminantes; y su influencia en las propiedades mecánicas. Para la etapa experimental usaron probetas de morteros de cemento convencional Tipo I y cemento blanco con el fin de realizar ensayos de resistencia, porosimetría, análisis térmico diferencial, ángulo de escurrimiento, ángulo de contacto y autolimpieza [2].
González [3], se enfocó en el uso del dióxido de titanio (TiO2), y su influencia en la resistencia a la compresión y su aspecto estético como material fotocatalítico para la fabricación de morteros mediante la elaboración de ensayos donde se sustituyó de manera gradual el cemento por dióxido de titanio en morteros [3]. El TiO2 es utilizado en la construcción usualmente en lechadas para evitar la absorción del polvo y suciedades en superficies en mesones de cocina o también la lechada entre cerámicas como es el baño, patios y también en la desinfección de aguas sometidos directamente con el exterior. Jiménez y Moreno, mencionaron que la construcción es un proceso más amigable con el medio ambiente y que es un desafío para nuestra sociedad. Al incorporar el fotocatalizador $\mathrm{TiO} 2$ desarrolla propiedades controladas por la luz solar para generar la purificación del aire y la auto-limpieza [4].

Variable independiente: Dióxido de titanio (TiO2). Porcentaje de dióxido de titanio: realizar distintas mezclas de morteros de cemento Pórtland Tipo I con diferentes porcentajes de dióxido de titanio. Evaluar el comportamiento del dióxido de titanio en la mezcla. Variable dependiente: Propiedades mecánicas y autolimpiantes. Propiedades mecánicas: ensayo de fluidez, ensayo de absorción del mortero, ensayo de resistencia a la compresión. Propiedades autolimpiantes: intemperismo y ensayo de rodamina.

Debe expresar en forma precisa el problema, el objetivo general, indicar el fundamento del estudio o proyecto realizado y la metodología empleada para su presentación. Se recomienda seguir el esquema adoptado en la revista Universidad, Ciencia y Tecnología: introducción, desarrollo (metodología, resultados y su discusión), las conclusiones y las referencias bibliográficas. Sustentar el aporte o contribución en base a una revisión breve del estado del arte, citando ordenadamente las referencias bibliográficas relacionadas con el tema. Para cerrar con el apartado, explicar en forma concisa la organización o estructura del artículo. Recuerde que el artículo completo debe tener una extensión máxima de 12 páginas. Evite el uso de citas textuales.

\section{Desarrollo}

El cemento Pórtland se fabrica a partir de materiales calcáreos (caliza) y arcillosos con alto contenido de sílice y alúmina, adicionalmente se agrega óxido de hierro para mejorar la composición química. En los estudios previos Wang, Zhang y Gao, demostraron que las nanopartículas de TiO2 pueden acelerar la hidratación del cemento y mejorar el desarrollo de resistencia de los materiales cementosos a temperatura ambiente. Sin embargo, todavía se desconoce el rendimiento de materiales cementosos que contienen nanopartículas de $\mathrm{TiO} 2$ a bajas temperaturas [5]. El cemento usado en la presente investigación es el cemento Portland Tipo I, destinado a usos como fabricación de concretos de mediana y alta resistencia a la compresión, preparación de concretos para elementos estructurales, fabricación de morteros para tarrajeos, etc., este cemento cumple con la NTP 334.009 y con la norma ASTM C-150 [6], [7]. El agua de mezclado es adicionada junto al cemento y agregados para la obtención de 
una pasta hidratada con una determinada fluidez que asegure la trabajabilidad de la mezcla, por lo tanto, a mayor cantidad de agua mayor fluidez de la pasta [8]. El dióxido de titanio ( $\mathrm{TiO} 2)$, es el óxido natural del titanio con característica opaco, estable, incombustible y no tóxico, que se suele comercializar en forma de polvo blanco. Tiene tres modificaciones cristalinas rutilo (tetragonal), anatasa (tetragonal) y brookita (ortorómbica). De estas tres formas cristalinas del TiO2, el rutilo es la más estable, ya que la anastasa y la brookita se transforman en rutilo bajo calentamiento. Los tipos rutilo y anastasa tienen más aplicaciones industriales [9].

El agregado fino es arena de origen natural o artificial que pasan el tamiz $9.5 \mathrm{~mm}$ (3/8”) quedando retenido en el tamiz $0.075 \mathrm{~mm}\left(\mathrm{~N}^{\circ} 200\right)$. Este material ocupa la mayor parte del volumen constituyente del mortero cuya fracción depende del diseño de acuerdo al uso que se le dará. Según la NTP 400.011, el agregado fino debe estar libre de cantidades perjudiciales de polvo, terrones, partículas escamosas o blandas, materia orgánica, sales u otras sustancias dañinas [6]. Los límites granulométricos dados por la NTP 399.607 no son aplicables para la arena fina [6]. Sin embargo, se realizó el ensayo para conocer el módulo de finura del agregado cuyos resultados son mostrados en la Tabla 1.

Tabla 1. Clasificación del agregado fino según el módulo de finura

\begin{tabular}{ll}
\hline Módulo de finura & Agregado fino \\
\hline Menor que 2.00 & Muy fino o extra fino \\
$2.00-2.30$ & Fino \\
$2.30-2.60$ & Ligeramente fino \\
$2.60-2.90$ & Mediano \\
$2.90-3.20$ & Ligeramente grueso \\
$3.20-3.50$ & Grueso \\
Mayor que 3.50 & Muy grueso o extra grueso \\
\hline
\end{tabular}

En el estudio efectuado por Chavarry, Chavarría, Valencia, Pereyra, Arrieta y Rengifo (2020), reforzaron un hormigón mediante la incorporación de vidrio molido para controlar la contracción plástica con distintas dosificaciones y coloración (verde y marrón), adoptando 4 tipos de mezcla ( $1 \%$ y $5 \%$ en relación al volumen del agregado fino), para una resistencia $\mathrm{f}^{\prime} \mathrm{c}=210 \mathrm{Kg} / \mathrm{cm} 2$, fueron ensayadas a compresión, flexión y fisuración [10]. El método empleado fue el deductivo, orientación aplicada, enfoque cuantitativo y como instrumento de recolección de datos retrolectivo, de tipo de investigación descriptivo, correlacional y explicativo. El diseño experimental, longitudinal, prospectivo y el estudio de cohorte (causa-efecto).

El presente estudio acoge la tipología empleada por Chavarry et. al dado que emplea un método deductivo, porque reconoce e identifica las variables de estudio, plantea la hipótesis correspondiente para cada uno de sus objetivos, operacionaliza las variables y propone un porcentaje óptimo de TiO2, es aplicada, ya que mejora las propiedades mecánicas y autolimpiantes del mortero, tiene un enfoque cuantitativo, toda vez que los resultados del estudio se van a presentar indicadores, porcentajes y costos por $\mathrm{m} 2$ de tarrajeo en fachadas con la incorporación de $\mathrm{TiO} 2$, el instrumento de recolección de datos es retrolectivo, porque la investigación utilizó formatos y/o fichas del laboratorio de materiales de la Universidad Ricardo Palma para organizar, recopilar, resumir, anotar la información y/o datos utilizados en el estudio [10].

\section{Metodología}

El estudio es de tipo descriptivo, correlacional y explicativo toda vez que busca la relación o grado de asociación exis- tente entre las variables estableciéndose la dosificación de los componentes del mortero con el porcentaje óptimo de $\mathrm{TiO} 2$. $\mathrm{El}$ diseño de la investigación fue experimental, longitudinal y prospectivo, debido a que se incorporó diferentes porcentajes de TiO2 (variable independiente). Por último, el estudio del diseño fue de cohorte (causa-efecto), porque estudia el efecto del TiO2 en las propiedades mecánicas y autolimpiantes del mortero.

Se diseñaron cuatro tipos de mezclas con $0 \%, 5 \%, 7.5 \%$ y $10 \%$ de TiO2 para el ensayo de absorción y 9 cubos de $50 \mathrm{~mm}$ de lado por cada mezcla de mortero de cemento Pórtland Tipo I. Para el ensayo de resistencia a la compresión se elaboraron 12 cubos de $50 \mathrm{~mm}$ de lado de mortero de cemento Portland Tipo I, por cada mezcla de los cuales se ensayaron 3 testigos a 1 día, 3 días, 7 días y 28 días. Para el ensayo de rodamina se elaboraron 3 cubos de $50 \mathrm{~mm}$ de lado por cada mezcla de mortero de cemento Pórtland Tipo I. Para la prueba de intemperismo se tarrajearon 4 paneles de $0.50 \mathrm{~m} \times 0.60 \mathrm{~m}$ con las distintas mezclas de mortero de cemento Pórtland Tipo I.

La técnica de investigación se basó en la observación del fenómeno y la recopilación de datos para su posterior análisis. Los principales instrumentos se desarrollaron mediante la realización de ensayos de laboratorio:

NTP 399.631: Método de ensayo normalizado para la tasa de absorción del agua de morteros de albañilería. NTP 334.003: Procedimiento para la obtención de pastas y morteros de consistencia plástica por mezcla mecánica. NTP 334.057: Método de ensayo para determinar la fluidez de morteros de cemento Pórtland.

NTP 334.051: Método de ensayo para determinar la resistencia a la compresión de morteros de cemento Pórtland 
usando especímenes cúbicos de $50 \mathrm{~mm}$ de lado. UNI 11259: Determinazione dell'attività fotocatalitica di liganti idraulici. Método de la rodamina. Seguimiento documentado fotográficamente para prueba de intemperismo [11].

Criterios de validez y confiabilidad de los instrumentos: Los ensayos de laboratorio fueron determinados por las normas técnicas peruanas, que definen las características de los equipos a utilizar. El laboratorio de ensayo de materiales de la Universidad Ricardo Palma cuenta con equipos calibrados de acuerdo a norma, es por esta razón que los instrumentos son confiables.

El ensayo de absorción se desarrolló según la norma NTP 399.631, se moldearon 9 cubos de $50 \mathrm{~mm}$ de lado de cada diseño de mezcla, que fue curado durante 28 días para luego proceder con el ensayo de absorción [6]. Se registró el peso seco de los cubos, luego se introdujeron dentro del recipiente con agua y se tomaron los pesos de la muestra ensayada en los intervalos de $0.25 \mathrm{~h}, 1.4 \mathrm{~h}$ y $24 \mathrm{~h}$ para hallar su absorción total.

El ensayo de fluidez se basó en la norma NTP 334.057, se realizaron mezclas de mortero según diseño, dichas mezclas fueron ensayadas en la mesa de flujo para obtener su porcentaje de fluidez. El ensayo de resistencia a la compresión se desarrolló según la norma NTP 334.0521, se moldearon 12 cubos de $50 \mathrm{~mm}$ de lado de cada diseño de mezcla, donde cada 3 cubos fueron curados durante 24 horas, 3, 7 y 28 días respectivamente para luego ser ensayados y obtener los valores de resistencia del mortero a la compresión (f'm) según edades de curado [6].

El ensayo de Rodamina B se realizó según la Norma Italiana UNI 11259 donde se impregnó una cara de los cubos de $50 \mathrm{~mm}$ de mortero con rodamina $\mathrm{B}$ y fueron expuestas a una lámpara de rayos UV, se registraron los valores en el sistema de color CIE mediante el uso de un colorímetro [12], [11]. Las mediciones se realizaron a las 4 horas y a las 26 horas para determinar el porcentaje de degradación de color.

El procesamiento de información fue de acuerdo a lo indicado en cada norma respectiva a cada ensayo ya que, cada uno tiene sus índices de medición. Los análisis de datos y comparación fueron obtenidos estadísticamente y mediante comparaciones fotográficas. Para contrastar la hipótesis, se realizó la prueba estadística de normalidad de Shapiro Wilk y para verificar la fiabilidad de los resultados mediante la prueba de ANOVA.

\section{Resultados}

La incorporación del 5\%, 7.5\% y 10\% de TiO2 en la mezcla de mortero disminuyó el porcentaje de fluidez en $10.32 \%$, $15.27 \%$ y $20.86 \%$ respectivamente en relación a la fluidez del mortero sin contenido de $\mathrm{TiO} 2$ debido a que la densidad del mortero se incrementa con la adición de $\mathrm{TiO} 2$, el mortero con $5 \%$ de $\mathrm{TiO} 2$ mantiene una consistencia plástica siendo adecuado para su trabajabilidad, por otro lado la resistencia a la compresión a la edad de 28 días en $7.16 \%, 11.13 \%$, $19.49 \%$ respectivamente, esto se debe a que el porcentaje de incorporación del fotocatalizador fue en reemplazo del volu- men absoluto del cemento. La tasa de absorción de agua a las 24 horas de exposición del mortero disminuyó en 11.33\%, $15.78 \%$ y $18.13 \%$ respectivamente, el resultado favorece a la durabilidad del mortero ante el intemperismo, las tasas de absorción a las $0.25,1.4$ y 24 horas de exposición de agua entre el mortero con $5 \%$ y $7.5 \%$ de $\mathrm{TiO} 2$ no tienen diferencias significativas, por lo tanto, la adición del $5 \%$ de $\mathrm{TiO} 2$ producirá los mismos efectos que el $7.5 \%$ de $\mathrm{TiO} 2$.

El ensayo de rodamina dio como resultado que el único mortero sin actividad fotocatalítica fue la que no contiene $\mathrm{TiO} 2$ debido a que no cumplió con lo estipulado en la norma UNI 11259 en los resultados de los factores de fotodegradación R4 y R26, Asimismo, no existen diferencias significativas entre el factor R26 del mortero con $7.5 \%$ de $\mathrm{TiO} 2 \mathrm{y} \mathrm{el}$ mortero con $10 \%$ de $\mathrm{TiO} 2$ [11]. El comportamiento de los paneles de mortero con $\mathrm{TiO} 2$ ante el intemperismo demostró la propiedad autolimpiante que adquiere el mortero al estar adicionado con $\mathrm{TiO} 2$ ya que, mantuvo su color inicial durante el tiempo de estudio y la absorción de agua de lluvia fue menor a comparación del panel sin contenido de TiO2 al igual que el tiempo de secado comprobando su característica hidrofílica e hidrófoba. El precio unitario del mortero por metro cuadrado sin $\mathrm{TiO} 2$ fue $\mathrm{S} / .27 .66$ y el mortero con $5 \%$ de $\mathrm{TiO} 2$ de S/.34.14. Sin embargo, el precio del acabado final de la fachada con mortero (espesor del tarrajeo $=1.5 \mathrm{~cm}$ ) convencional pintado es $\mathrm{S} / .40 .11$.

No existe literatura concerniente a estudios del TiO2, enfocada a la degradación de $\alpha$-metiltestosterona por fotocatálisis de TIo2. La aplicación del $\mathrm{TiO} 2$ como fotocatalizador utilizando radiación solar es su limitada absorción de menos del $5 \%$ de su espectro. Poco acceso a los ensayos de rodamina e intemperismo.

El diseño de mezcla propuesto por el presente estudio fue el que tiene una incorporación de 5\% TiO2, con el uso de un aditivo plastificante para mejorar la trabajabilidad de la mezcla. La resistencia a la comprensión disminuyó en 7.16\%, cuando se le incorporó un 5\%, disminuyendo más aun aumentando la dosificación de $\mathrm{TiO} 2$, con respecto al costo con un $5 \% \mathrm{TiO} 2$, se incrementó un $23.43 \%$ con respecto al tradicional para Jiménez y Moreno detallan que la resistencia a la compresión con 5\% de $\mathrm{TiO} 2$ se incrementa hasta en $6 \%$, mientras que en las muestras de $7.5 \%$ y $8.5 \%$ disminuye por el aumento de porosidad; según el análisis de costos, el mortero con $5 \%$ de $\mathrm{TiO} 2$ en toda la capa del mortero, este tiene un incremento del $32 \%$ con respecto al tradicional [4]. Abella (2015), concluye que la adición de $\mathrm{TiO} 2$ no disminuye la resistencia y proporciona al mortero propiedades autolimpiantes mediante la exposición a la radiación UV; asimismo, los morteros de cemento convencional presentaron reducción de porosidad. Para Grebenişan, Szilagyi, Hegyi, Mircea y Baeră, con respecto al costo manifiestan que el uso de estos materiales para la construcción de edificios conduce a una reducción efectiva de los costos en lo que respecta al mantenimiento, lavado y reparación de edificios, descontaminación y esterilización e incluso a la posible prevención de olores urbanos desagradables. La tasa de absorción de agua a las 24 horas de 
exposición del mortero con 5\%, 7.5\% y 10\% de TiO2 disminuyó en $11.33 \%, 15.78 \%$ y $18.13 \%$ respectivamente en relación al mortero sin TiO2 [13].

Medina, concluyó que, el uso del dióxido de titanio disminuye el porcentaje de absorción del mortero, su resistencia a la compresión y el porcentaje de fluidez. La incorporación del $5 \%, 7.5 \%$ y $10 \%$ de $\mathrm{TiO} 2$ en la mezcla de mortero disminuye el porcentaje de fluidez en $10.32 \%, 15.27 \%$ y $20.86 \%$ respectivamente debido a que la densidad del mortero se incrementa con la adición de TiO2 [1]. El mortero con 5\% de $\mathrm{TiO} 2$ mantiene una consistencia plástica siendo adecuado para su trabajabilidad, para Kaszynskam y Olczyk , indican que los estudios realizados han demostrado que la adición del dióxido de titanio influye en la fluidez de la pasta de cemento porque aumenta significativamente la velocidad del proceso de fraguado. El ensayo de rodamina dio como resultado que el único mortero sin actividad fotocatalítica fue la que no contiene TiO2 debido a que no cumplió con lo estipulado en la norma UNI 11259 en los resultados de los factores de fotodegradación R4 y R26 [14]. El comportamiento de los paneles de mortero con $\mathrm{TiO} 2$ ante el intemperismo demuestra la propiedad autolimpiante ya que, mantuvo su color inicial durante el tiempo de estudio y la absorción de agua de lluvia fue menor a comparación del panel sin contenido de $\mathrm{TiO} 2$ al igual que el tiempo de secado comprobando su característica hidrofílica e hidrófoba. El uso de fotocatalizadores puede ser beneficioso para los elementos concretos y el entorno circun- dante, González indica que los cementos modificados con fotocatalizadores pueden ser empleado para diseñar materiales para la construcción amigables con el medio ambiente ya que degradan contaminantes y compuestos orgánicos que pueden deteriorar la parte estética de las estructuras [3].

Realizar pruebas de descontaminación del aire a las muestras de mortero para medir la eficacia del material fotocatalítico ante la eliminación de $\mathrm{COVs}$, $\mathrm{NOx}$ y $\mathrm{CO} 2$ siguiendo los procedimientos establecidos en las normas XP B44-013 y UNI 11247. Realizar un estudio de la actividad antibacteriana de las muestras de mortero con contenido de $\mathrm{TiO} 2$ mediante el ensayo descrito en la norma JIS R 1702.

Para calcular el porcentaje mínimo que hace que el mortero adquiera propiedades fotocatalíticas es recomendable realizar ensayos con muestras de mortero con porcentajes menores al 5\% de $\mathrm{TiO} 2$. El mortero con contenido de $\mathrm{TiO} 2$ como revestimiento es el acabado final de la fachada, para continuar con la investigación se sugiere realizar ensayos de fotodegradación de color con muestras de mortero coloreados.

El ensayo indica el porcentaje de aumento del diámetro de la base de un cono truncado de mortero compactado, cuando éste es colocado sobre una mesa de flujo, dejándola caer desde una altura de $12.7 \mathrm{~mm} 25$ veces en un tiempo de 15 segundos. Los resultados obtenidos del ensayo se muestran en la Figura 1.

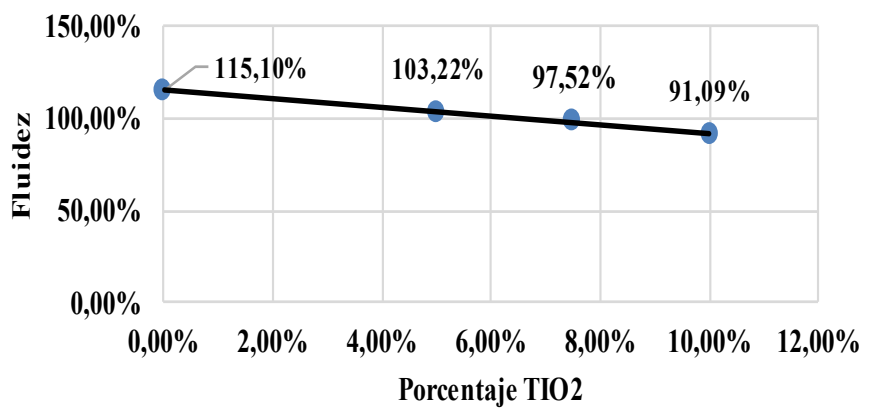

Fig. 1. Fluidez de cada tratamiento de mortero según porcentaje de TiO2.

La incorporación del 5\%, 7.5\% y 10\% de TiO2 en la mezcla de mortero disminuyó el porcentaje de fluidez en $10.32 \%$, $15.27 \%$ y $20.86 \%$ respectivamente en relación a la fluidez del mortero sin contenido de $\mathrm{TiO} 2$, debido a que la densidad del mortero se incrementa con la adición de TiO2. El mayor porcentaje de fluidez obtenido fue $115.10 \%$ que pertenece al mortero sin adición y el menor porcentaje fue $91.09 \%$ obtenido del mortero con 10\% de TiO2. El mortero con 5\% de $\mathrm{TiO} 2$ mantiene una consistencia plástica siendo adecuado para su trabajabilidad. Asimismo, las mezclas de mortero con $7.5 \%$ y $10 \%$ de $\mathrm{TiO} 2$ continuaron siendo trabajable a pesar que el porcentaje de fluidez de cada uno indica que tiene una consistencia dura.

Se moldearon 12 cubos para cada mezcla de mortero con un distinto porcentaje de $\mathrm{TiO} 2$, las edades de ensayo fueron 24 horas, 3, 7 y 28 días para cada 3 especímenes. La aplicación de la carga en la máquina de ensayo se realizó en las caras del espécimen que estaban en contacto con las superficies planas del molde. Los resultados obtenidos de los ensayos se muestran en las Figuras 1 y 2. 


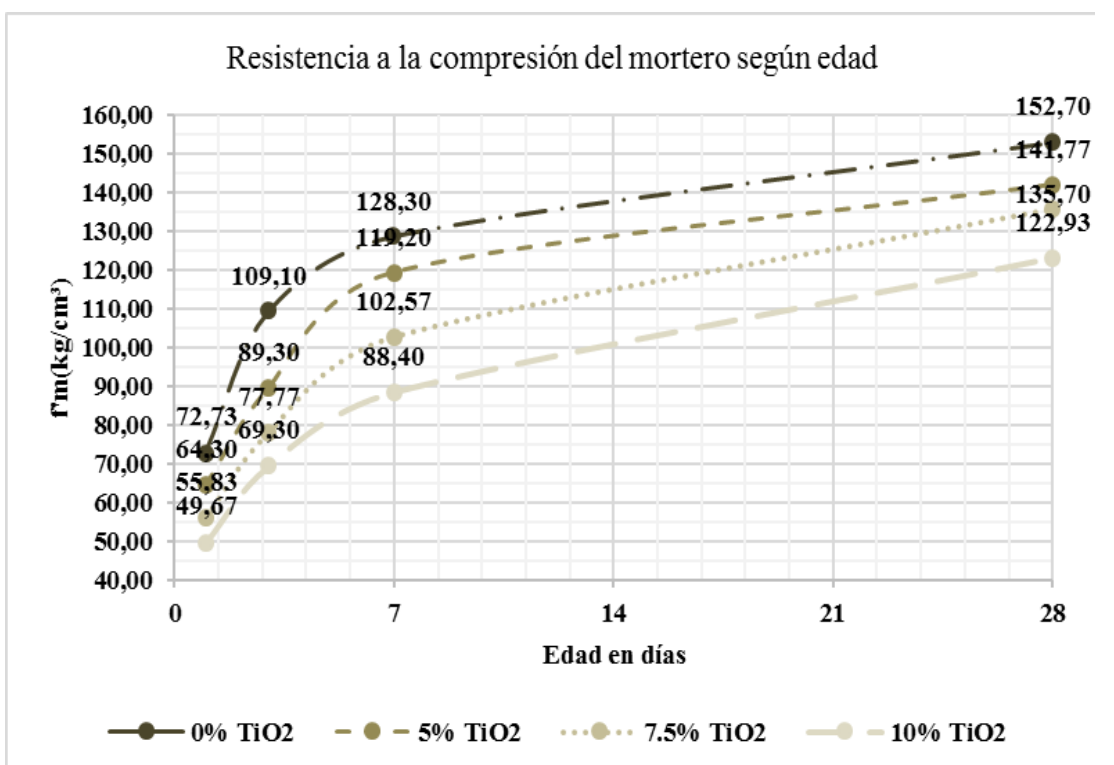

Fig. 2. Resistencia a la compresión del mortero según la edad y las dosificaciones en porcentaje de TiO2 (a).

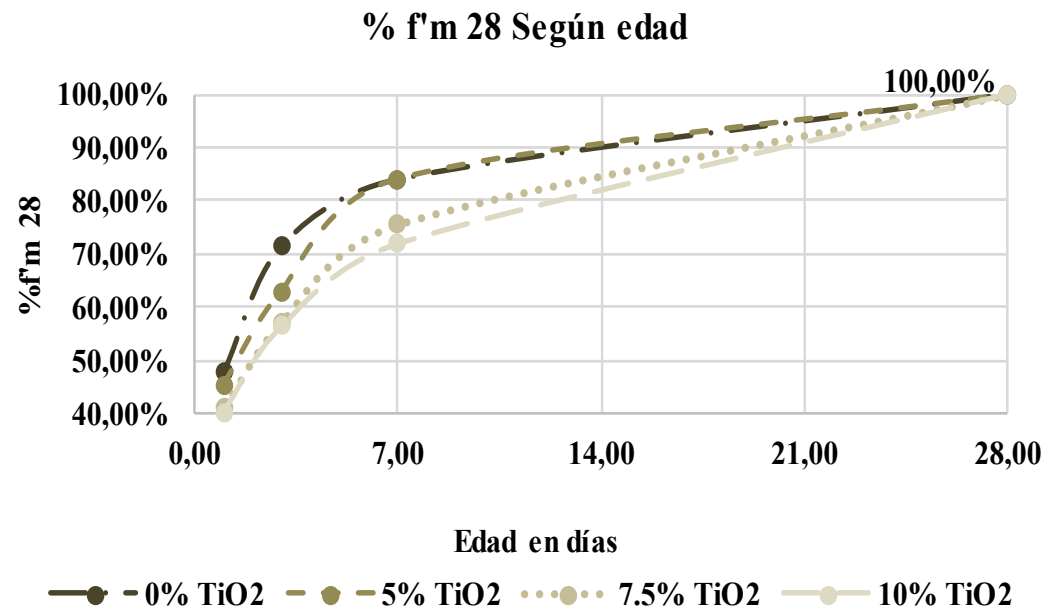

Fig. 3. Porcentaje de f'm según la edad del mortero y las dosificaciones en porcentajes de TiO2 (b).

La adición del 5\%,7.5\% y $10 \%$ de $\mathrm{TiO} 2$ en el mortero disminuyó la resistencia a la compresión a la edad de 28 días en $7.16 \%, 11.13 \%, 19.49 \%$ respectivamente en relación al mortero sin $\mathrm{TiO} 2$, esto se debe a que el porcentaje de incorporación del fotocatalizador fue en reemplazo del volumen absoluto del cemento. La mayor resistencia a la compresión obtenida a la edad de 28 días $\left(152.70 \mathrm{~kg} / \mathrm{cm}^{2}\right)$ fue del mortero sin $\mathrm{TiO} 2$ y la menor resistencia $\left(122.93 \mathrm{~kg} / \mathrm{cm}^{2}\right)$ del mortero con $10 \%$ de $\mathrm{TiO} 2$. Asimismo, el endurecimiento del mortero con $7.5 \%$ y $10 \%$ de $\mathrm{TiO} 2$ en edades tempranas fue lento a comparación del mortero sin adición. El desarrollo relativo de resistencia del mortero con $5 \%$ de $\mathrm{TiO} 2$ como un porcentaje de la resistencia a los 28 días fue parecido al del mortero sin contenido de $\mathrm{TiO} 2$.

Para este proyecto se moldearon 9 cubos de mortero para cada uno de los porcentajes de $\mathrm{TiO} 2$, los cuales fueron ensayados cada 3 especímenes a $0.25,1.4$ y 24 horas. El cálculo de la tasa de absorción de agua (AT) en gramos $/ 100 \mathrm{~cm}^{2}$ para cada periodo de tiempo (T) y cada espécimen se aplica la fórmula (1):

$$
A T=\frac{(W T-W 0) \times 10000}{L 1-L 2}
$$

Donde: $\mathrm{WT}=$ Peso del espécimen en el tiempo $\mathrm{T}$ en gramos. $\mathrm{W} 0=$ Peso inicial del espécimen en gramos. $\mathrm{L} 1=\mathrm{La}$ longitud promedio de la superficie del cubo del espécimen en $\mathrm{mm}$. L2 = El ancho promedio de la superficie del cubo del espécimen en $\mathrm{mm}$. 


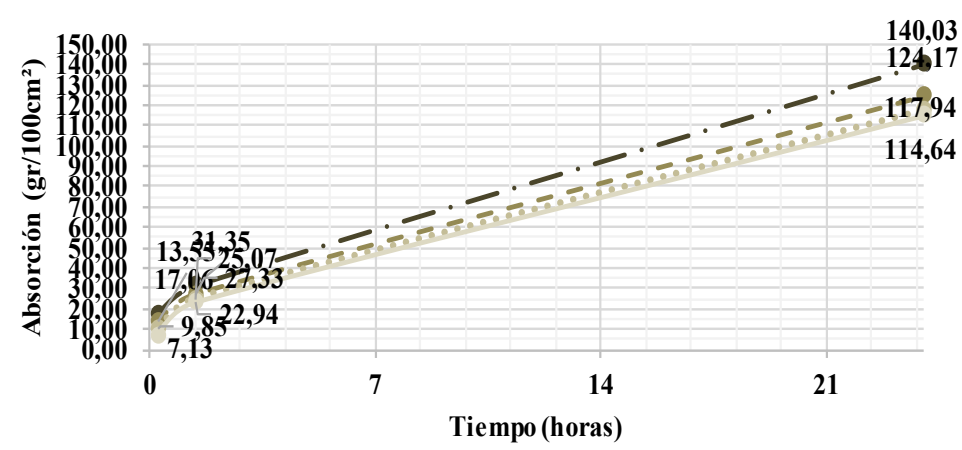

$\longrightarrow-0 \% \mathrm{TiO}--5 \% \mathrm{TiO} 2 \ldots 0 \cdots 7.5 \% \mathrm{TiO} \longrightarrow 10 \% \mathrm{TiO} 2$

Fig. 4. Tasa de absorción de agua del mortero según los distintos porcentajes de TiO2 absorción del mortero según tiempo de exposición al agua (a).

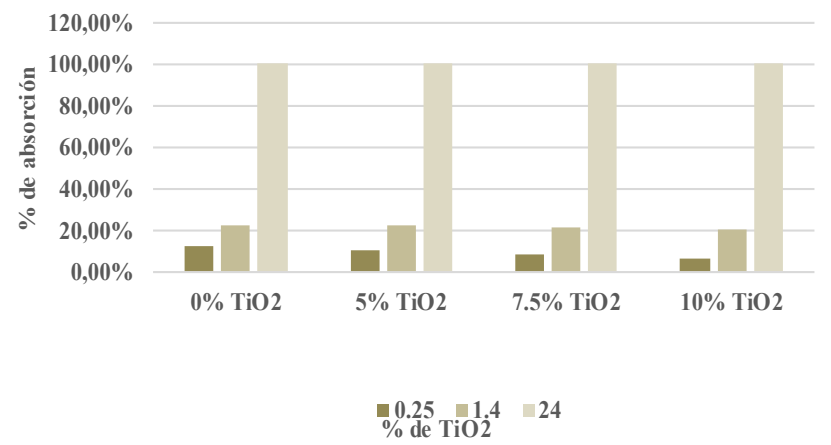

Fig. 5. Porcentaje de tasa de absorción de agua $24 \mathrm{~h}$ del mortero (b).

La tasa de absorción de agua a las 24 horas de exposición del mortero con 5\%, 7.5\% y 10\% de TiO2 disminuyó en $11.33 \%, 15.78 \%$ y $18.13 \%$ respectivamente en relación al mortero sin $\mathrm{TiO} 2$, el resultado favorece a la durabilidad del mortero ante el intemperismo. La mayor tasa de absorción fue $140.03 \mathrm{gr} / 100 \mathrm{~cm}^{2}$ obtenido del mortero sin adición y la menor tasa de absorción de agua fue $114.64 \mathrm{gr} / 100 \mathrm{~cm}^{2}$ que pertenece al mortero con $10 \%$ de $\mathrm{TiO} 2$. A las 0.25 horas de exposición al agua, el mortero con $\mathrm{TiO} 2$ absorbe en menor medida relativa como porcentaje de la tasa de absorción a las 24 horas con respecto al mortero sin $\mathrm{TiO} 2$. Asimismo, la tasa de absorción a las $0.25,1.4$ y 24 horas de exposición de agua entre el mortero con $5 \%$ y $7.5 \%$ de $\mathrm{TiO} 2$ no tienen diferencias significativas, por lo tanto, la adición del 5\% de TiO2 producirá los mismos efectos que el 7.5\% de TiO2.

El ensayo de rodamina dio como resultado que el único mortero sin actividad fotocatalítica fue la que no contiene TiO2 debido a que no cumple con lo estipulado en la norma UNI 11259 en los resultados de los factores de fotodegradación R4 y R26 [11]. A partir del mortero con 7.5\% de TiO2 el proceso de fotodegradación se desaceleró considerablemente ya que, la fotocatálisis se realiza superficialmente por lo tanto una mayor adición de $\mathrm{TiO} 2$ es innecesaria. Asimismo, no existen diferencias significativas entre el factor R26 del mortero con $7.5 \%$ de $\mathrm{TiO} 2$ y el mortero con $10 \%$ de $\mathrm{TiO} 2$.

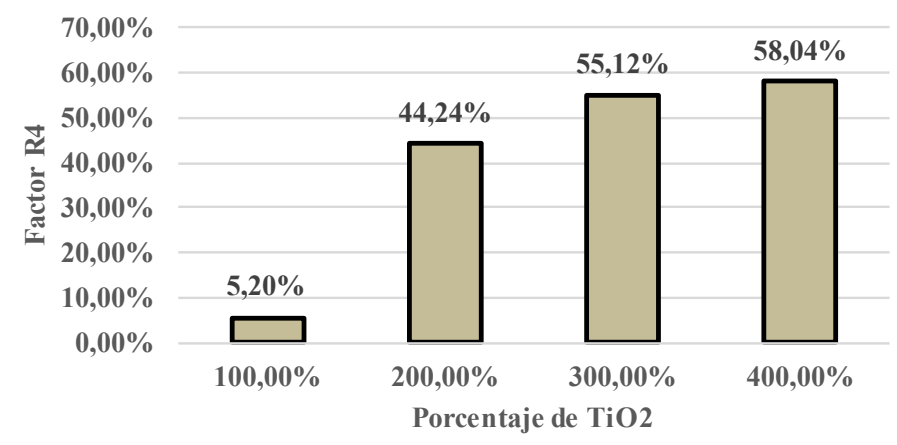

Fig. 6. Factor de Fotodegradación R4, color del mortero con distintos porcentajes de TiO2 (4 horas) (a). 


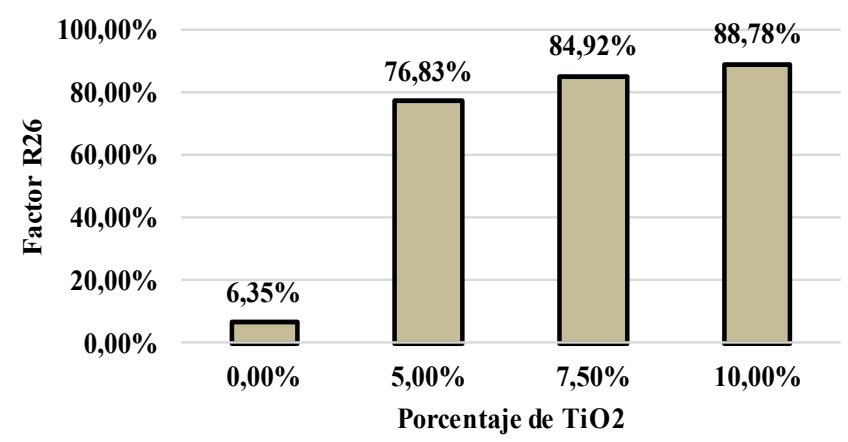

Fig. 7. Fotodegradación R26 de color del mortero con distintos porcentajes de TiO2 (26 horas) (b).

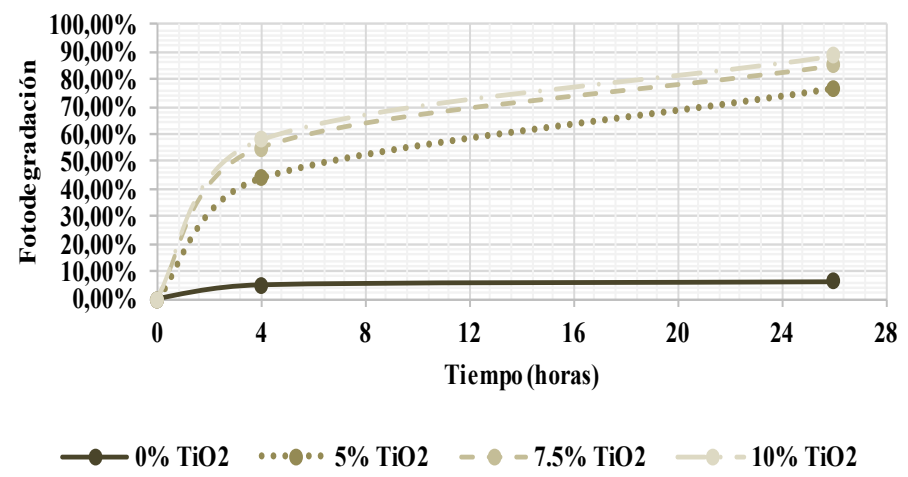

Fig. 8. Fotodegradación del mortero según tiempo de exposición a rayos UV (c).

Para evaluar cualitativamente el comportamiento fotocatalítico de los morteros de cemento Pórtland Tipo I con distintos porcentajes de $\mathrm{TiO} 2$, se construyeron 4 paneles los cuales fueron expuestos al medio ambiente durante 3 meses con el fin de observar día a día su respuesta ante el intemperismo. Se observa que el color blanquecino de los morteros con contenido de $\mathrm{TiO} 2$ se mantiene, el panel sin contenido de $\mathrm{TiO} 2$ presenta adherencia de partículas contaminantes que se muestra a través de manchas. La presencia de lluvias en el segundo mes muestra la diferencia de comportamiento del panel sin tratamiento de $\mathrm{TiO} 2$ con los paneles que si contienen TiO2. Se observa que la absorción capilar es mayor en el panel de $0 \% \mathrm{TiO} 2$ y menor en el panel de $10 \% \mathrm{TiO} 2$ siendo este menos permeable. Se observa que el color de los paneles de $5 \%, 7.5 \%$ y $10 \%$ de contenido de $\mathrm{TiO} 2$ se mantienen limpios a pesar de estar en contacto con el piso lo contrario al panel sin contenido de $\mathrm{TiO} 2$ el cual muestra manchas por adhesión de partículas contaminantes.

Se realizó un análisis de costos unitarios de cada uno de los morteros con $0 \%, 5 \%, 7.5 \%$ y $10 \%$ de $\mathrm{TiO} 2$ para determinar la viabilidad económica. Se incluyó el análisis de costos unitarios de pintura por $\mathrm{m} 2(\mathrm{~S} / .12 .45)$, para comparar el tarrajeo de muros exteriores con mortero convencional con un acabado final de pintado con respecto al tarrajeo con morteros con contenido de TiO2. Los resultados se muestran en la tabla 2.

Tabla 2. Costo por $\mathrm{m} 2$ de tarrajeo de fachada con mortero de $1,5 \mathrm{~cm}$ de espesor con diferentes

\begin{tabular}{lllll}
\hline $\begin{array}{l}\text { Mortero } \\
\text { TiO2 }\end{array}$ & $\begin{array}{l}\text { Costo unitario } \\
\text { (tarrajeo m2) }\end{array}$ & $\begin{array}{l}\text { Costo unitario } \\
\text { (tarrajeo }+ \text { pintura } \\
\mathbf{m 2})\end{array}$ & $\begin{array}{l}\text { Costo } \\
\text { unitario }\end{array}$ & $\begin{array}{l}\text { Incremento } \\
\text { de costo }\end{array}$ \\
& S/. & Sotal & $\mathbf{( \% )}$ \\
& & 12.45 & S/. & \\
\hline $\mathbf{0 \%}$ & 27.66 & 0 & 40.11 & $0.00 \%$ \\
$\mathbf{5 . 0 0 \%}$ & 34.14 & 0 & 34.14 & $23.43 \%$ \\
$\mathbf{7 . 5 0 \%}$ & 37.38 & 0 & 37.38 & $35.14 \%$ \\
$\mathbf{1 0 . 0 0 \%}$ & 40.61 & & 40.61 & $46.82 \%$ \\
\hline
\end{tabular}


El análisis de precios unitarios dio como resultado que si el acabado final de la fachada con mortero sin $\mathrm{TiO} 2$ es $\mathrm{S} /$. 27.66, comparándolo con los precios unitarios del mortero con adición del $5 \% \mathrm{TiO} 2$ se incrementa en un $23.43 \%$ con respecto al tradicional, siendo entre estos el más económico el mortero con $5 \%$ de $\mathrm{TiO} 2$ con un costo por $\mathrm{m} 2$ de $\mathrm{S} / .34 .14$. Sin embargo, si el acabado final de la fachada con mortero convencional es pintado el precio total de $\mathrm{S} / .40 .11$.

\section{Conclusiones}

El comportamiento de los paneles de mortero con 5\%, $7.5 \%$ y $10 \%$ de $\mathrm{TiO} 2$ ante el intemperismo demostró la propiedad autolimpiante que adquiere el mortero al estar adicionado con $\mathrm{TiO} 2$ ya que, mantuvo su color inicial durante el tiempo de estudio y la absorción de agua de lluvia fue menor a comparación del panel sin contenido de $\mathrm{TiO} 2$ al igual que el tiempo de secado comprobando su característica hidrofílica e hidrófoba. El hormigón autolimpiante, es una alternativa eficaz para proporcionar un medio ambiente más limpio que contribuya a la sostenibilidad y a un medio ambiente verde. Se mejorarán las prestaciones mecánicas del hormigón autolimpiante mediante la adición de materiales fotocatalíticos bajo luz UV y la degradación fotocatalítica del contaminante gaseoso describe que el hormigón autolimpiante representa un material que contiene nanomateriales, es decir, nanopartículas $\mathrm{TiO} 2$ con propiedades fotocatalíticas, de un compuesto que contiene $\mathrm{TiO} 2-\mathrm{SiO} 2$, exhiben un efecto superhidrófilo sobre las superficies, es decir, en presencia de radiación UV, el agua que llega a estas superficies tiene la capacidad de formar películas que se apoderan de las impurezas con mayor facilidad [9], [13].

\section{Referencias}

[1]E. Medina and H. Pérez, "Influencia del fotocatalizador dióxido de titanio en las propiedades autolimpiables y mecánicas del mortero de cemento - arena 1:4 - Cajamarca," Universidad Nacional de Cajamarca, 2017.

[2]G. Abella, "Mejora de las propiedades de materiales a base de cemento que contienen $\mathrm{TiO} 2$ : propiedades autolimpiantes," Universidad Politécnica de Madrid, 2015.

[3]J. Gonzalez, "El Dióxido de titanio como material fotocatalitico y su influencia en la resistencia a la compresión en Morteros," Universidad de San Buenaaventura Seccional Be1lo, 2015.

[4]D. Jimenez and J. Moreno, "Efecto del reemplazo de cemento portland por el dioido de titanio en las propiedades mecanicas del mortero," Pontificia Universidad Javeriana, 2016.

[5]L. Wang, H. Zhang, and Y. Gao, "Effect of TiO2 nanoparticles on physical and mechanical properties of cement at low temperatures," Adv. Mater. Sci. Eng., 2018, doi: $10.1155 / 2018 / 8934689$.

[6]Comisión de Normalización y de Fiscalización de Barreras Comerciales no Arancelares, Norma Técnica Peruana. Perú, 2013, p. 29.

[7]ASTM Internacional, “ASTM C150,” 2021. https://www.
astm.org/Database.Cart/Historical/C150-07-SP.htm.

[8]M. Issa, "( current astm c150 / aashto m85 ) with limestone and process addition ( ASTM C465 / AASHTO M327) on the performance of concrete for pavement and Prepared By," 2014.

[9]S. Zailan, N. Mahmed, M. Abdullah, A. Sandu, and N. Shahedan, "Review on characterization and mechanical performance of self-cleaning concrete," MATEC Web Conf., vol. 97, pp. 1-7, 2017, doi: 10.1051/matecconf/20179701022.

[10]C. Chavarry, L. Chavarría, A. Valencia, E. Pereyra, J. Arieta, and C. Rengifo, "Hormigón reforzado con vidrio molido para controlar grietas y fisuras por contracción plástica," Pro Sci., vol. 4, no. 31, pp. 31-41, 2020, doi: 10.29018/issn.2588-1000vol4iss31.2020pp31-41.

[11]D. Tobaldi, "Materiali ceramici per edilizia con funzionalità fotocatalitica," Università di Bologna, 2009.

[12]Norme UNI, "Norma Italiana UNI 11259," 2016. http:// store.uni.com/catalogo/uni-11259-2008?josso_back_to=http://store.uni.com/josso-security-check.php\&josso_cmd=login_optional\&josso_partnerapp_host=store.uni.com.

[13]E. Grebenisan, H. Szilagyi, A. Hegyi, C. Mircea, and C. Baera, "Directory lines regarding the desing and production of self-cleaning cementitious composites," Sect. Green Build. Technol. Mater., vol. 19, no. 6, 2019.

[14]M. Kaszynska, "The influence of TIO2 nanoparticles on the properties of self-cleaning cement mortar," Int. Multidiscip. Sci. GeoConference SGEM, pp. 333-341, 2018.

\section{RESUMEN CURRICULAR}

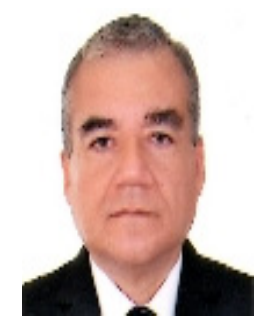

Carlos Chavarry, Doctor en ingeniería Civil, Maestro en Gestión, Dirección y Diseño de proyectos, diseño Asesor en proyectos con la aplicación de Metodologías como BIM, LC, PMBOK, SCRUM, LAST PLANNER. Docente Universitario de Posgrado en Gestión de Proyectos y Metodología de la Investigación. Gerente, Residente y supervisorde obra.

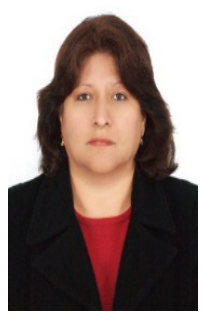

Liliana Chavarria, Candidata a Doctora en Ingeniería Civil, Maestra en Gerencia de la Construcción Moderna, Ex Jefa del Laboratorio de Ensayo de Materiales de Escuela Profesional de Ingeniería Civil (EPIC) Universidad Ricardo PalmaMiembro de las Normas Técnicas Peruanas en Agregados y Concreto, Miembro del Instituto Americano del Concreto ACI Perú. 


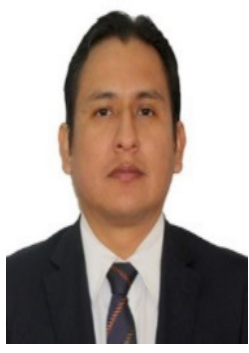

Xavier Laos, Consultor y Docente Universitario, Ingeniero Civil, Doctor En Ingeniería Civil, Candidato a Maestro en Dirección de la Construcción UPC, Maestro en Administración UPC, Management Program - Harvard. Experiencia JIT, Dirección de la Construcción, B2B y Gestión Comercial Retail. Miembro del Instituto Americano del Concreto - ACI Perú.

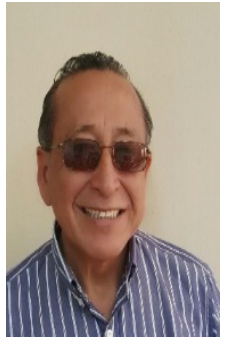

Andrés Valencia, Doctor en educación, Maestro en Gerencia de la Construcción moderna, Decano de la facultad de Ingeniería Civil-Universidad Nacional Federico Villarreal, miembro de la comisión de acreditación de la EPIC-FIA de la Universidad San Martín de Porres, Vicerrector Académico de la Universidad Nacional de Moquegua). Docente de pre grado en Cálculo diferencial e integral y Formulación de Proyectos de Ingeniería Civil.

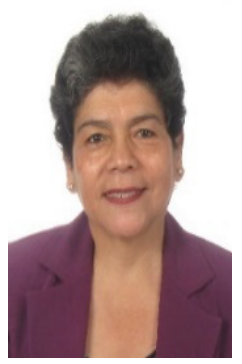

Enriqueta Pereyra, Candidata a Doctora en Ingeniería Civil, Maestra en Gerencia de la Construcción, Ex Directora de la Escuela Profesional de Ingeniería Civil (EPIC) Universidad Ricardo Palma. Miembro de las Normas Téenicas Peruanas en Agregados y Concreto, Miembro del Instituto Americano del Concreto ACI Perú.

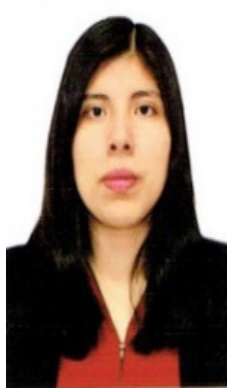

Karen Martínez, Ingeniera Civil, Bachiller en Arquitectura (título en trámite de sustentación), estudios de Maestría en Ciencias con Mención en Ingeniería Estructural (UNI). Diplomado en Arquitectura Bioc limática. Diplomado Ingeniería Sís mica y el Cálculo y el Diseño Estructural Aplicado a Edificios. Residente y supervisora de obras retail. 